\title{
Direct Chemical Vapor Deposition of Graphene
}

\author{
on Dielectric Surfaces
}

Ariel Ismach, Clara Druzgalski, Samuel Penwell, Max Zheng, Ali Javey, Jeffrey Bokor

and Yuegang Zhang

AUTHOR EMAIL ADDRESS: yzhang5@lbl.gov

\section{Supporting Information}

\section{Methods}

Electron-beam evaporator (Edwards) was used to deposit copper (99.999\%, Alfa Aesar) on dielectric substrates (ST-cut quartz Hoffman Materials LLC; Si wafers Addison Engineering Inc.; m-plane sapphire Monocrystal PLC and fused silica). Then the samples were placed in a tube furnace (Lindberg Blue) and pumped to 100-500 mTorr. $35 \mathrm{sccm}$ of $\mathrm{H}_{2}$ (99.999\% Airgas) was flowed during the heating to the CVD temperature, $1000{ }^{\circ} \mathrm{C}$. The growth was done by flowing a mixture of $\mathrm{H}_{2}$ (2 sccm) and $\mathrm{CH}_{4}$ (35 sccm- 99.99\% Airgas). The system was cooled down under a flow of $35 \mathrm{sccm} \mathrm{H}_{2}$. The samples were characterized by Raman spectroscopy and imaging (Witec with a $532 \mathrm{~nm}$ green laser), scanning electron microscopy (SEM- Zeiss Gemini Ultra-55), energy dispersive X ray spectroscopy (EDX), optical microscopy and atomic force microscopy (AFM- Tapping mode; Digital Instruments 3000). 


\section{EDX Characterization}

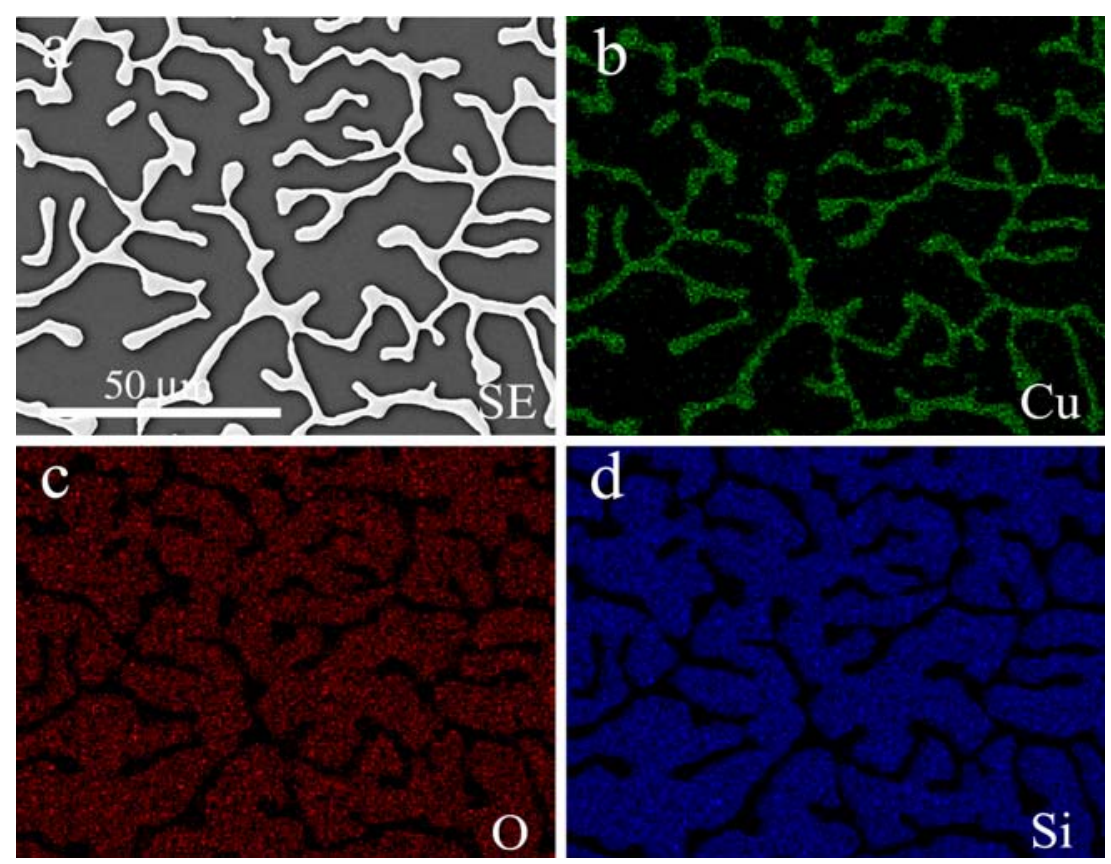

Figure SI1: EDX characterization. (a) Secondary electrons imaging of $\mathrm{Cu}$ fingers on quartz, white, after 2 hour CVD growth. The deposited metal thickness was $450 \mathrm{~nm}$. (b)-(d) Elemental Elemental mapping of $\mathrm{Cu}, \mathrm{O}$ and $\mathrm{Si}$, respectively. The reproduced finger shape in (b) is very clear, confirming the absence of metal between the fingers. 


\section{Raman Characterization}

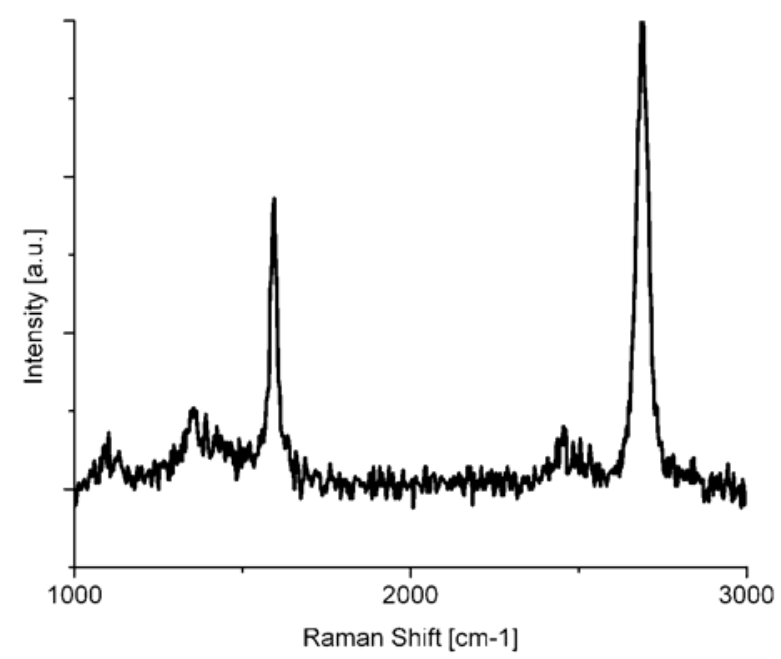

Figure SI2: Graphene growth on copper foil. Raman spectra of a graphene layer growth on $\mathrm{Cu}$ foil and transferred to glass. The spectra suggest a high quality layer $[\mathrm{I}(\mathrm{G}) / \mathrm{I}(2 \mathrm{D})<1$ and the FWHM $\left.\sim 34 \mathrm{~cm}^{-1}\right]$. The graphene was grown similar to the conditions in ref 19 in the letter. 

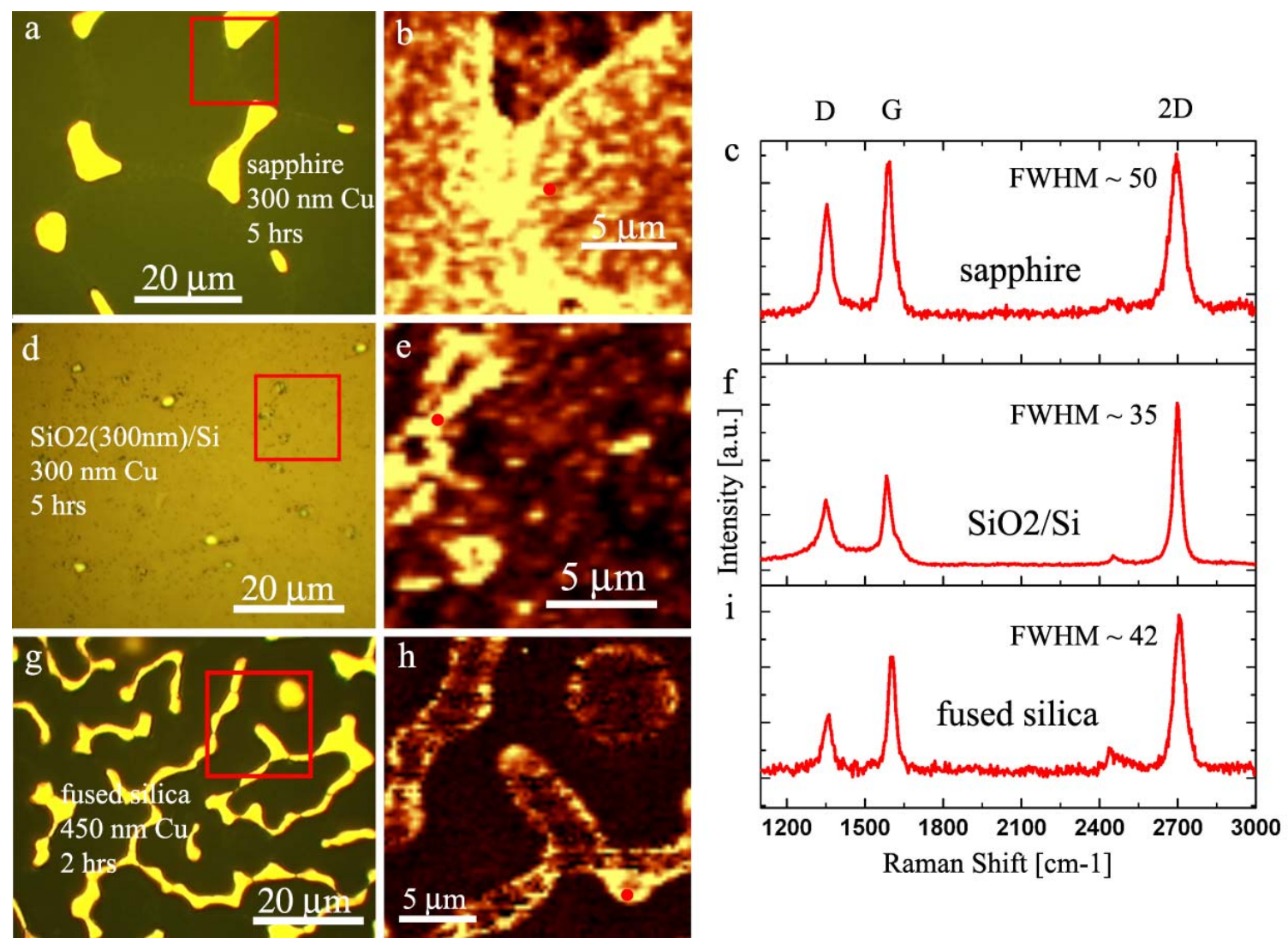

Figure SI3: Additional Raman characterization images. Optical, Raman mapping with the 2D band, and Raman spectra of graphene growth on three different substrates. (a)-(c) M-plane sapphire; (d)-(f) $\mathrm{SiO}_{2} / \mathrm{Si}$ wafer and (g)-(h) fused silica. Only on sapphire the graphene is shown to be continuous (b). The discontinuity may arise from the de-wetting and evaporation of the metal during temperature ramping-up, prior to the CVD. The Raman spectra in (c), (f) and (i) is normalized with the G band. 

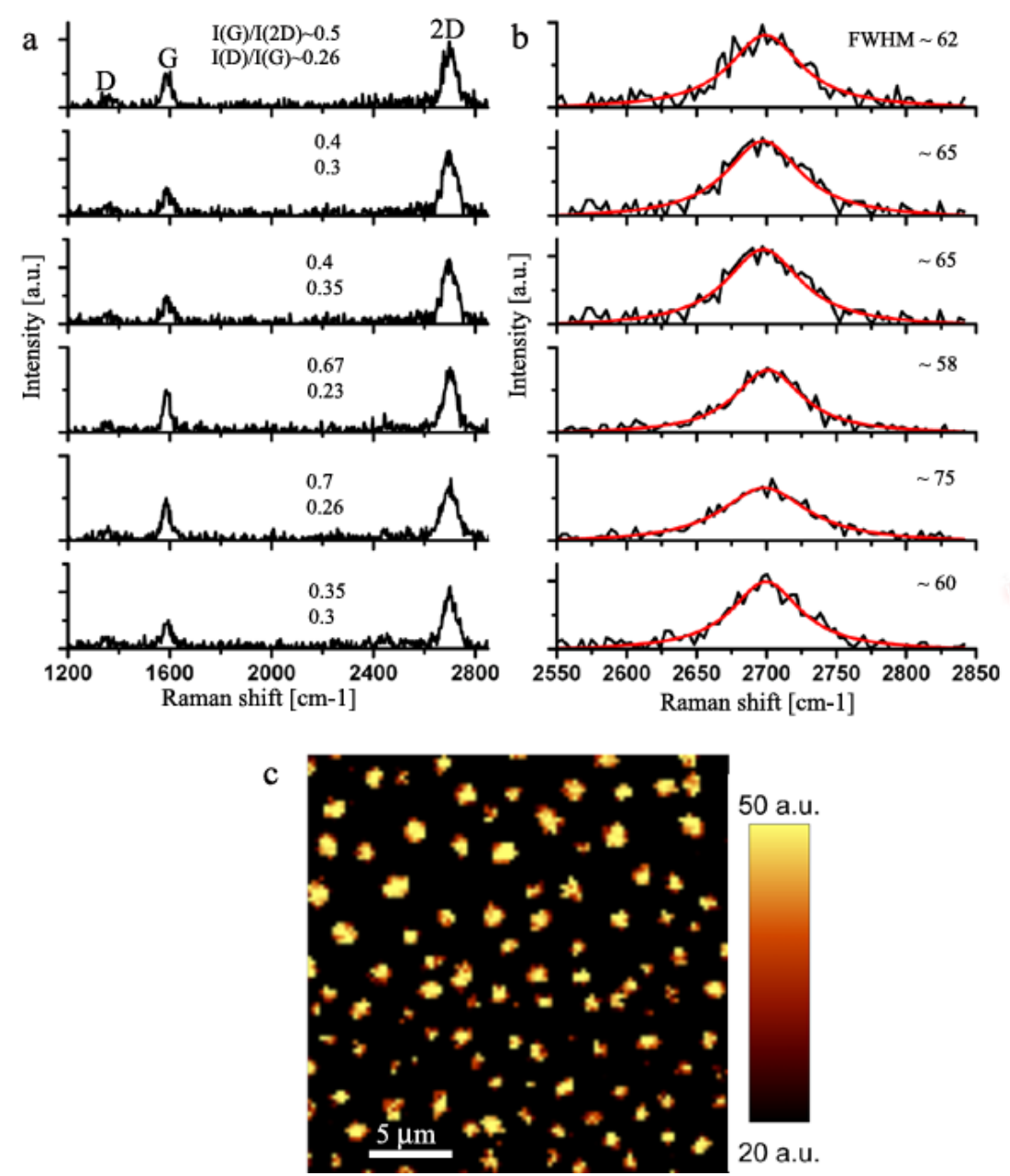

Figure SI4: Additional Raman characterization of the sample in Figure 4. (a) Raman spectra from six different dots in Figure 4c, showing the $I(G) / I(2 D)$ and $I(D) / I(G)$ ratios for every spectrum. (b) Lorentzian curve fitting to the 2D band in every spectrum and its FWHM value. (c) FWHM mapping of the same sample in Figure 4c. 


\section{De-wetting / evaporation evolution with CVD time}

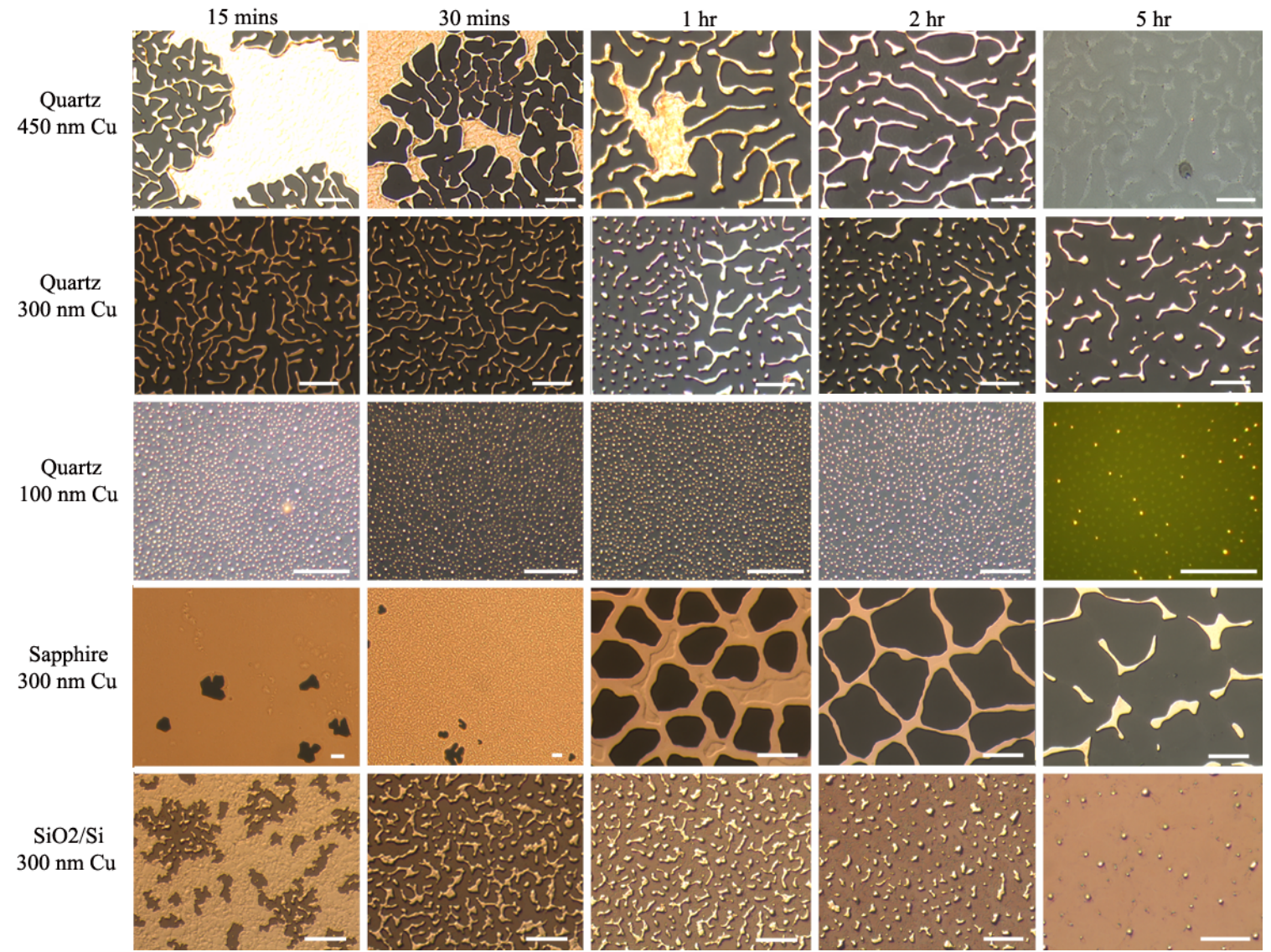

Figure SI5: De-wetting evolution as a function of CVD time, top, and substrate type, left. The scale bar is $20 \mu \mathrm{m}$ in all images. The de-wetting behavior for the different substrates can be appreciated, as well as the influence of the metal film thickness (first three rows). In some cases (quartz with 100 and 300nm Cu thick film) the films breaks into fingers or dots after few minutes of heating. In thicker layers on quartz and $300 \mathrm{~nm} \mathrm{Cu}$ on sapphire and $\mathrm{SiO}_{2} / \mathrm{Si}$ the films breaks completely only after 2 hrs of CVD. There is no intermediate state between the continuous films and the fingers, suggesting a two stage process: The first is a fast transition into finger or dot-shape and the second, the completely de-wetting and evaporation of the fingers or dots. The top right optical image is after 7 hrs CVD growth, all the rest of the column corresponds to images of samples after 5 hrs growths as specified. 


\section{Additional AFM and SEM characterization}

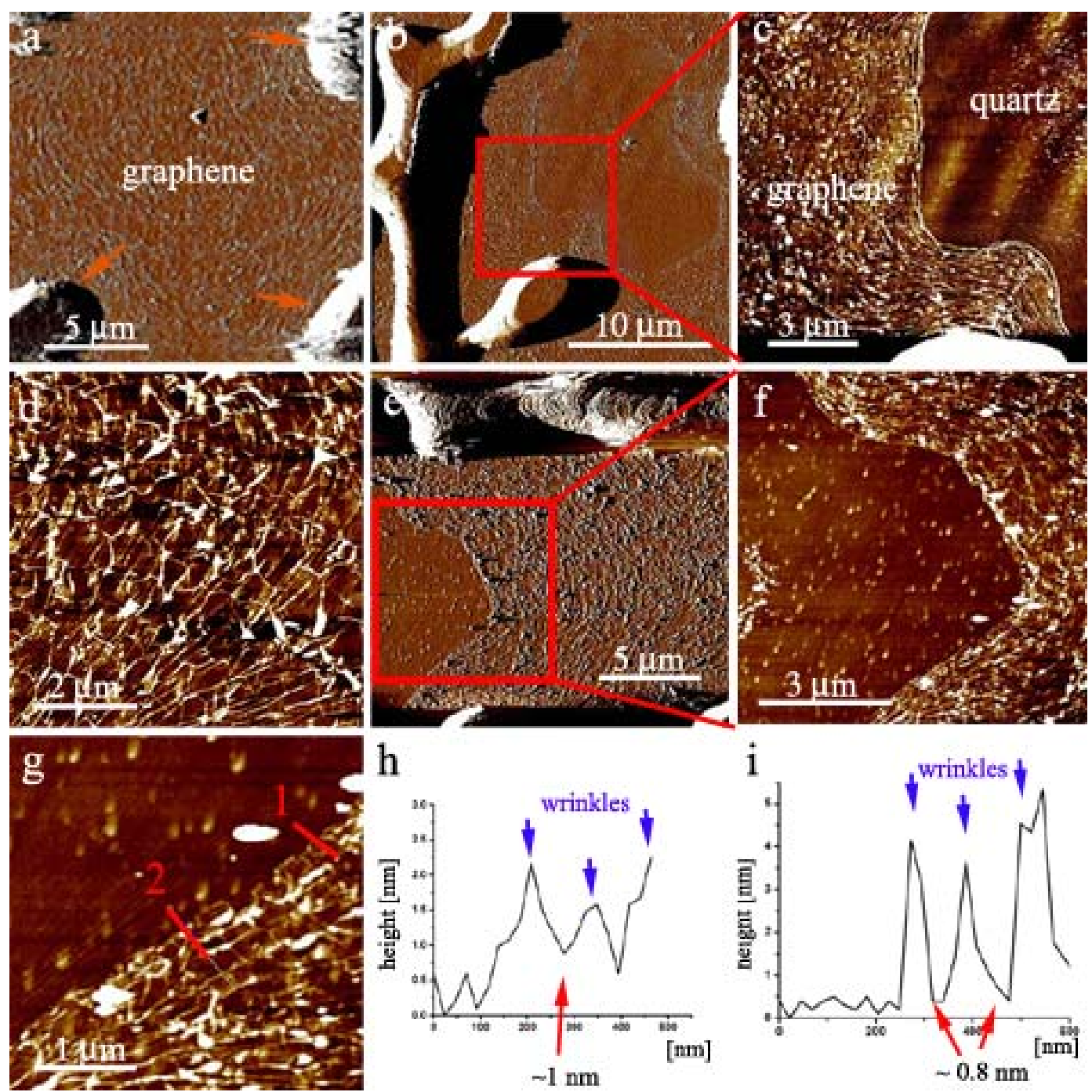

Figure SI6. Additional AFM images. (a) and (b) show amplitude images of continuous and discontinuous graphene layer, respectively. SEM imaging with the in-lens detector of large area covered with graphene. (c) Height image of the red square area in (b). (d) Height image of a continuous graphene showing the quasi-periodic structure. (e) Amplitude and (f) height image of the red square in (e). (g) Height image showing a graphene edge. (h) and (i) section analysis along the red lines \#1 and \#2, respectively, showing layer heights of 1 and $0.8 \mathrm{~nm}$, corresponding to $\sim 1-2$ graphene layers. 

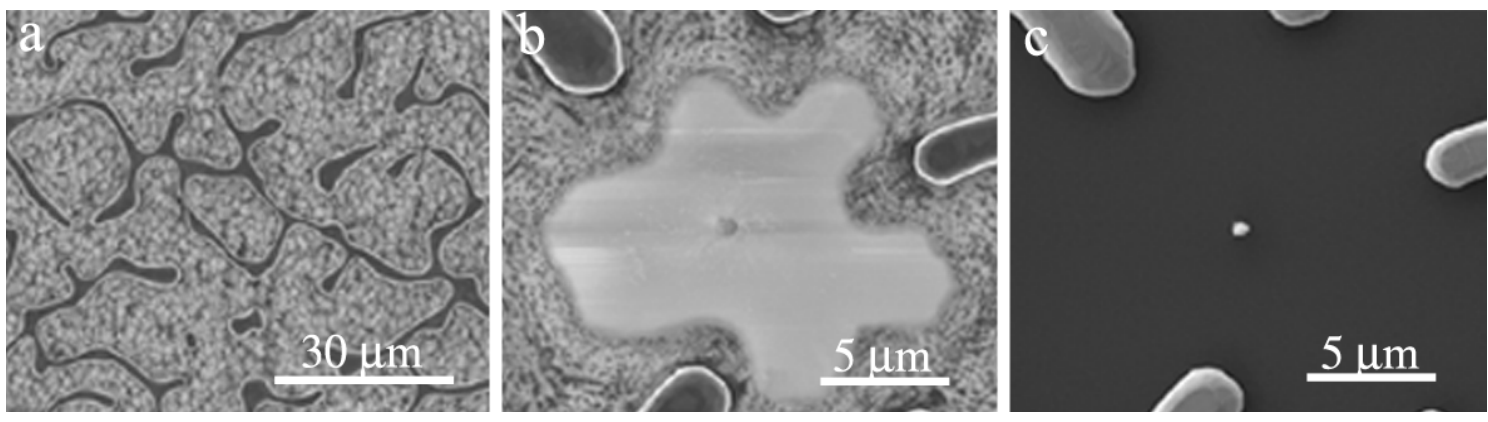

Figure SI7. Additional SEM images. (a) SEM imaging with the in-lens detector of large area covered with graphene. (b) and (c) SEM imaging using the in-lens and secondary electron (SE) detector respectively. The wrinkle structure of the graphene is very clear in (h) but not seen in (i), indicating that the graphene layer, despite its wrinkle structure, is flat and is hardly detected by the topography-sensitive SE detector. 